Corrigendum: Classification and characterization of microsatellite instability across 18 cancer types

Ronald J Hause, Colin C Pritchard, Jay Shendure \& Stephen J Salipante

Nat. Med. 22, 1342-1350 (2016); published online 3 October 2016; corrected after print 19 July 2017

In the version of this article initially published, in Figure 4d, the column labels UCED and STAD were inadvertently switched. The error has been corrected in the HTML and PDF versions of the article.

\title{
Corrigendum: Targeting CASP8 and FADD-like apoptosis regulator ameliorates nonalcoholic steatohepatitis in mice and nonhuman primates
}

Pi-Xiao Wang, Yan-Xiao Ji, Xiao-Jing Zhang, Ling-Ping Zhao, Zhen-Zhen Yan, Peng Zhang, Li-Jun Shen, Xia Yang, Jing Fang, Song Tian, Xue-Yong Zhu, Jun Gong, Xin Zhang, Qiao-Fang Wei, Yong Wang, Jing Li, Lu Wan, Qingguo Xie, Zhi-Gang She, Zhihua Wang, Zan Huang \& Hongliang Li

Nat. Med. 23, 439-449 (2017); published online 20 February 2017; corrected after print 26 July 2017

In the version of this article initially published, the authors inadvertently left out information in the Online Methods section regarding a second injection of AAV8-CFLAR(S1) 7 weeks after the first injection in the monkey experiments to ensure stable expression of CFLAR(S1) in the livers of the monkeys that received the injections. This correction does not change any results or conclusions of the paper. The error has been corrected in the HTML and PDF versions of the article.

\section{Erratum: Thermoneutral housing exacerbates nonalcoholic fatty liver disease in mice and allows for sex-independent disease modeling}

Daniel A Giles, Maria E Moreno-Fernandez, Traci E Stankiewicz, Simon Graspeuntner, Monica Cappelletti, David Wu, Rajib Mukherjee, Calvin C Chan, Matthew J Lawson, Jared Klarquist, Annika Sünderhauf, Samir Softic, C Ronald Kahn, Kerstin Stemmer,

Yoichiro Iwakura, Bruce J Aronow, Rebekah Karns, Kris A Steinbrecher, Christopher L Karp, Rachel Sheridan, Shiva K Shanmukhappa, Damien Reynaud, David B Haslam, Christian Sina, Jan Rupp, Simon P Hogan \& Senad Divanovic

Nat. Med.; doi:10.1038/nm.4346; corrected online 21 June 2017

In the version of this article initially published online, a grant supporting the authors' work was omitted from the Acknowledgments section. The grant "NIH T32AI118697 (associated with D.A.G.)" has now been added. The error has been corrected in the print, PDF and HTML versions of this article.

\section{Erratum: Elimination of large tumors in mice by mRNA-encoded bispecific antibodies}

Christiane R Stadler, Hayat Bähr-Mahmud, Leyla Celik, Bernhard Hebich, Alexandra S Roth, René P Roth, Katalin Karikó,

Özlem Türeci \& Ugur Sahin

Nat. Med. 23, 815-817 (2017); published online 12 June 2017; corrected after print 20 July 2017

In the version of this article initially published, in the "Polymer/lipid-based formulation of mRNA" section of the Online Methods, the text incorrectly stated that mRNA was in $190 \mathrm{ml}$ rather than $190 \mu \mathrm{l}$ of cold DMEM. The error has been corrected in the HTML and PDF versions of the article. 